\title{
Produtividade e qualidade pós-colheita de cebola adubada com doses crescentes de nitrogênio e potássio
}

\author{
Geraldo M de Resende; Nivaldo Duarte Costa; José Maria Pinto \\ Embrapa Semi-Árido, C. Postal 23, 56302-970 Petrolina-PE; gmilanez@cpatsa.embrapa.br
}

\section{RESUMO}

O experimento foi conduzido de março a setembro de 2000, em campo experimental da Embrapa Semi-Árido, em Petrolina-PE com o objetivo de avaliar a influência de doses de nitrogênio e potássio sobre as características produtivas e qualidade pós-colheita da cebola (Allium cepa L.). Utilizou-se a cultivar Franciscana IPA-10 e o delineamento experimental de blocos ao acaso, em esquema fatorial 4 x 3, compreendendo quatro doses de nitrogênio $(0 ; 60 ; 120$ e 180 $\left.\mathrm{kg} \mathrm{ha}^{-1}\right)$ e três doses de potássio $\left(0 ; 90\right.$ e $180 \mathrm{~kg} \mathrm{ha}^{-1}$ de $\left.\mathrm{K}_{2} \mathrm{O}\right)$, com três repetições. A produtividade comercial apresentou comportamento linear na ausência da adubação potássica, enquanto as doses de 90 e $180 \mathrm{~kg} \mathrm{ha}^{-1}$ de potássio proporcionaram máximas produtividades nas doses de 175,8 e 169,4 $\mathrm{kg} \mathrm{ha}^{-1}$ de nitrogênio. Observou-se com o aumento das doses de nitrogênio uma redução gradativa na produção de bulbos refugos (não comerciais). Na ausência da adubação potássica obteve-se um efeito linear em função das doses de nitrogênio para massa fresca do bulbo. Para as doses de 90 e $180 \mathrm{~kg} \mathrm{ha}^{-1}$ de potássio, o maior rendimento de massa fresca do bulbo foi obtido com a dose de 153,3 $\mathrm{kg} \mathrm{ha}^{-1}$ de nitrogênio. A classificação de bulbos comerciais de cebola foi influenciada pelas doses de nitrogênio, sendo que a maior porcentagem de bulbos da classe $3(85,8 \%)$ correspondeu à dose de $153,6 \mathrm{~kg} \mathrm{ha}^{-1}$ de nitrogênio. Não se constatou efeitos significativos aos 20 dias para perda de massa, evidenciando-se efeitos significativos apenas aos 40 e 60 dias após cura (DAC), e não houve interação entre as doses de nitrogênio e potássio.

\begin{abstract}
Yield and post-harvest quality of onion fertilized with increasing levels of nitrogen and potassium

This study was carried out from March to September 2000, in Petrolina, Pernambuco State, Brazil, to evaluate the influence of nitrogen and potassium levels on yield characteristics and post-harvest quality of onion bulbs (Allium cepa L.). The cultivar Franciscana IPA10 was used. The experimental design was a randomized complete block in a $4 \times 3$ factorial scheme, involving four levels of nitrogen $(0$; $60 ; 120$ and $180 \mathrm{~kg} \mathrm{ha}^{-1}$ ) and three levels of potassium (0; 90 and 180 $\mathrm{kg} \mathrm{ha}^{-1}$ ) with three replications. The commercial yield showed a linear effect in the absence of potassium fertilization, while the levels of 90 and $180 \mathrm{~kg} \mathrm{ha}^{-1}$ of potassium provided maximum yield with the levels of 175.8 and $169.4 \mathrm{~kg} \mathrm{ha}^{-1}$ of nitrogen. Increasing the doses of nitrogen resulted in a reduction of the non-commercial yield (culls). In the absence of potassium fertilization, a linear effect was obtained as a result of the levels of nitrogen for fresh mass of the bulb. For the levels of 90 to $180 \mathrm{~kg} \mathrm{ha}^{-1}$ of potassium, the highest yield of fresh mass of the bulb was obtained with the level of $153.3 \mathrm{~kg} \mathrm{ha}^{-1}$ of nitrogen. The levels of nitrogen influenced the classification of commercial bulbs of onion and the largest percentage of bulbs of the class $3(85,8 \%)$ corresponded the dose of $153,6 \mathrm{~kg} \mathrm{ha}^{-1}$ of nitrogen. No significant mass loss occurred after 20 days and only after 40 and 60 days after cure did any significant effects become evident and there was no interaction between the levels of nitrogen and potassium.
\end{abstract}

Palavras-chave: Allium cepa, fertilização, rendimento, conservação pós-colheita.
Keywords: Allium cepa, fertilization, yield, postharvest conservation.

(Recebido para publicação em 30 de maio de 2007; aceito em 23 de junho de 2008)

A produção mundial de cebola em 2005 , foi de 59,5 milhões de toneladas, cultivadas em 3,2 milhões de hectares, o que proporcionou uma produtividade média de 18,6 t ha $^{-1}$ (FAO, 2007). No Brasil, a cebola ocupa entre as hortaliças o terceiro lugar em importância econômica (Souza \& Resende, 2002). Em 2006, a produtividade média nacional de acordo com o IBGE (2006) se situou em torno de 20,4 $\mathrm{tha}^{-1}$, sendo que nos estados de Pernambuco e Bahia, maiores produtores do Nordeste, houve uma produtividade média de 18,9 e 24,8 $\mathrm{t} \mathrm{ha}^{-1}$, respectivamente.

Os elementos absorvidos em maiores quantidades na matéria seca da cebola são o potássio e nitrogênio
(Filgueira, 2003). A absorção de nutrientes pela cebola cv. Pukekohe Early até a colheita foi de $218 \mathrm{~kg} \mathrm{ha}^{-1}$ de N, $204 \mathrm{~kg}$ $\mathrm{ha}^{-1}$ de K, $32 \mathrm{~kg} \mathrm{ha}^{-1}$ de P, $54 \mathrm{~kg} \mathrm{ha}^{-1}$ de $\mathrm{S}, 108 \mathrm{~kg} \mathrm{ha}^{-1}$ de Ca e $13 \mathrm{~kg} \mathrm{ha}^{-1} \mathrm{de} \mathrm{Mg}$ (Sher, 1996).

Quanto à capacidade de resposta dessa cultura às doses de nitrogênio, diversos autores relatam que este nutriente contribui marcadamente para a produtividade. $\mathrm{O}$ diâmetro do bulbo e a produtividade aumentaram com o incremento das doses até $80 \mathrm{~kg} \mathrm{ha}^{-1}$ de N, não havendo diferenças significativas quando da aplicação da dose de $120 \mathrm{~kg} \mathrm{ha}^{-1}$ de $\mathrm{N}$ nas condições da Índia (Singh \& Sharma, 1991). Farias et al. (1992) identificaram a dose de $115 \mathrm{~kg} \mathrm{ha}^{-1}$ para as condições do Vale do São Francisco, Petrolina-PE; enquanto Vachchani \& Patel (1996), na Índia, não verificaram diferenças entre as doses de 100 e 150 $\mathrm{kg} \mathrm{ha}^{-1}$ de N, para massa fresca de bulbo e produtividade, apesar de a dose de $150 \mathrm{~kg} \mathrm{ha}^{-1}$ ter proporcionado maior altura de planta, número de folhas e tamanho do bulbo.

Singh \& Dhankhar (1991) verificaram em bulbos armazenados sob condições ambientais que a perda de massa e a incidência de bulbos podres e brotados aumentaram com o incremento das doses de nitrogênio (80 a $160 \mathrm{~kg} \mathrm{ha}^{-1} \mathrm{de}$ $\mathrm{N})$ e reduziram com a dose de $100 \mathrm{~kg}$ $\mathrm{ha}^{-1}$ de $\mathrm{K}_{2} \mathrm{O}$. As doses de $80 \mathrm{~kg} \mathrm{ha}^{-1}$ de $\mathrm{N}$ mais $100 \mathrm{~kg} \mathrm{ha}^{-1}$ de $\mathrm{K}_{2} \mathrm{O}$ resultaram nas 
melhores respostas em qualidade de armazenamento. Estudando dez cultivares em condições de armazenamento refrigerado $\left(5^{\circ} \mathrm{C}\right)$ e ambiente $\left(25-30^{\circ} \mathrm{C}\right)$, Warid et al. (1996), observaram, após 25 semanas, que as perdas de massa por bulbos podres e brotados e perdas de água foram maiores no armazenamento em condições ambientais. Segundo os mesmos autores, a média de bulbos comerciais variou de 5,5 a 69,0\% em condições ambiente e 30,0 a 92,5\% sob refrigeração.

Embora a cebola seja uma cultura que extrai grandes quantidades de potássio do solo, nem sempre as respostas a esse nutriente, têm sido observadas (Filgueira, 2003). Segundo Shukla et al. (1992), a aplicação de $40 \mathrm{~kg} \mathrm{ha}^{-1}$ de $\mathrm{K}_{2} \mathrm{O}$ aumentou a produtividade, porém, não houve efeito adicional quando se utilizou as doses de 80 e $160 \mathrm{~kg} \mathrm{ha}^{-1}$, respectivamente. Por outro lado, Mohanty \& Das (2001) observaram aumento do diâmetro e da massa fresca do bulbo com a dose de $60 \mathrm{~kg} \mathrm{ha}^{-1}$ de $\mathrm{K}_{2} \mathrm{O}$, nas condições da Índia.

Nesse trabalho, procurou-se avaliar o efeito de doses de nitrogênio e potássio na produtividade e qualidade póscolheita de bulbos de cebola nas condições do Vale do São Francisco.

\section{MATERIAL E MÉTODOS}

O experimento foi conduzido de março a setembro de 2000, no Campo Experimental de Bebedouro, da Embrapa Semi-Árido, em Petrolina-PE. O solo classificado como Latossolo Vermelho Amarelo (Embrapa, 1999), apresentou: $\mathrm{pH}\left(\mathrm{H}_{2} \mathrm{O}\right)=6,6 ; \mathrm{Ca}=2,0 \mathrm{cmol}_{\mathrm{c}}$ $\mathrm{dm}^{-3} ; \mathrm{Mg}=0,7 \mathrm{cmol}_{\mathrm{c}} \mathrm{dm}^{-3} ; \mathrm{Na}=0,01$ $\mathrm{cmol}_{\mathrm{c}} \mathrm{dm}^{-3} ; \mathrm{K}=0,25 \mathrm{cmol}_{\mathrm{c}} \mathrm{dm}^{-3}$; $\mathrm{P}($ Mehlich $)=9,0 \mathrm{mg} \mathrm{dm}^{-3}$ e $\mathrm{MO}=6,8 \mathrm{~g}$ $\mathrm{kg}^{-1}$.

O delineamento experimental utilizado foi blocos ao acaso, em esquema fatorial $4 \times 3$, compreendendo quatro doses de nitrogênio $(0 ; 60 ; 120$ e 180 $\left.\mathrm{kg} \mathrm{ha}^{-1}\right)$ e três doses de potássio (0; $90 \mathrm{e}$ $180 \mathrm{~kg} \mathrm{ha}^{-1}$ ) com três repetições. A unidade experimental constituiu-se de um canteiro com oito linhas de $3,0 \mathrm{~m}$ de comprimento, espaçadas de $0,15 \mathrm{~m}$, com $0,10 \mathrm{~m}$ entre plantas, perfazendo uma área total 3,6 m² (3,0 x 1,2 m). Foram utilizadas como área útil as seis linhas centrais, retirando-se $0,50 \mathrm{~m}$ em cada extremidade $\left(1,80 \mathrm{~m}^{2}\right)$.

As adubações nitrogenada e potássica foram divididas em três parcelas, sendo a primeira realizada no plantio (1/3) e o restante (2/3) em duas coberturas aos 25 e 50 dias após transplante, sendo usadas como fontes a uréia e cloreto de potássio, aplicadas lateralmente às linhas de plantio. A adubação fosfatada $\left(135,0 \mathrm{~kg} \mathrm{ha}^{-1} \mathrm{P}_{2} \mathrm{O}_{5}\right)$ foi aplicada totalmente no plantio, de acordo com a análise de solo (Cavalcanti, 1998).

A semeadura da cultivar Franciscana IPA-10 foi feita em 09/03/00 e o transplante efetuado 30 dias após. O preparo do solo constou de aração, gradagem e levantamento dos canteiros a 0,20 m de altura. A cultura foi mantida sem plantas daninhas através de capinas manuais e a irrigação por microaspersão foi realizada três vezes por semana, com lâminas em torno de $10 \mathrm{~mm}$, baseada na evaporação do tanque classe $\mathrm{A}$, e suspensa 20 dias antes da colheita. Os demais tratos fitossanitários foram realizados de acordo com as recomendações para a cultura da cebola.

A colheita foi realizada em 20/07/ 00 quando as plantas apresentaram sinais avançados de senescência, como amarelecimento e seca das folhas e mais de $70 \%$ das plantas encontravam-se estaladas. A cura foi realizada por três dias ao sol e por 12 dias à sombra, em galpão ventilado.

Aos 15 dias após a cura foram avaliadas a produtividade de bulbos comerciais (bulbos perfeitos e com diâmetro transversal acima de $35 \mathrm{~mm}$ ) e não comerciais (refugos, com diâmetro inferior a $35 \mathrm{~mm}$ ) expressas em $\mathrm{t} \mathrm{ha}^{-1}$ ); a massa fresca de bulbo ( $\mathrm{g} \mathrm{bulbo}^{-1}$ ) foi determinada dividindo-se a massa de bulbos comerciais pelo número de bulbos. Após o período de cura, os bulbos foram armazenados em sacos de polipropileno à temperatura ambiente (temperatura média mensal $24,3^{\circ} \mathrm{C}$ e umidade relativa média de $60,6 \%$ ) e realizadas pesagens aos 20; 40 e 60 dias, sendo os valores comparados àqueles obtidos ao final da cura (15 dias após colheita). Os valores foram transformados em porcentagem de perda de mas- sa. A classificação de bulbos comerciais segundo o diâmetro transversal (mm) foi feita de acordo com Brasil (1995) em classe 2 (>35 até $50 \mathrm{~mm}$ de diâmetro); classe 3 (>50 até $70 \mathrm{~mm})$; classe 4 (>70 até $90 \mathrm{~mm})$ e classe 5 (>90 $\mathrm{mm})$. Os dados foram expressos em porcentagem.

Os dados coletados foram submetidos à análise de variância e regressão polinomial ao nível de $5 \%$ de probabilidade. Os dados de porcentagem foram transformados em arco-seno $\sqrt{P / 100}$ para efeitos de análise (Gomes, 2000).

\section{RESULTADOS E DISCUSSÃO}

Os resultados evidenciaram efeitos significativos independentes para as doses de nitrogênio e potássio, assim como para a interação entre estes fatores, dependendo das características avaliadas.

O efeito da ausência da adubação potássica sobre a produtividade comercial foi linear, enquanto as doses de 90 e $180 \mathrm{~kg} \mathrm{ha}^{-1}$ de potássio proporcionaram máximas produtividades nas doses de 175,8 e $169,4 \mathrm{~kg} \mathrm{ha}^{-1}$ de nitrogênio. Respostas positivas da aplicação de nitrogênio na cultura da cebola tem sido relatadas até a dose de $150 \mathrm{~kg} \mathrm{ha}^{-1}$ de $\mathrm{N}$ (Dias-Perez et al., 2003; Singh et al., 2004); assim como até as doses de 40 $\mathrm{kg} \mathrm{ha}^{-1}$ (Shukla et al., 1992) e $120 \mathrm{~kg}$ ha $^{-1}$ de $\mathrm{K}_{2} \mathrm{O}$ (Singh \& Verma, 2001). Já Baloch et al. (1991) verificaram maiores produtividades de bulbos associando $125 \mathrm{~kg} \mathrm{ha}^{-1}$ de $\mathrm{N}$ e $75 \mathrm{~kg} \mathrm{ha}^{-1}$ de $\mathrm{K}_{2} \mathrm{O}$; Syed et al. (2000) com $120 \mathrm{ha}^{-1}$ de $\mathrm{N}$ e $90 \mathrm{~kg} \mathrm{ha}^{-1}$ de $\mathrm{K}_{2} \mathrm{O}$; Lee et al. (2003), com $136 \mathrm{ha}^{-1}$ de $\mathrm{N}$ e $96 \mathrm{~kg} \mathrm{ha}^{-1} \mathrm{~kg}$ de $\mathrm{K}_{2} \mathrm{O}$; enquanto Mandira \& Khan (2003), obtiveram melhores rendimentos com 150 $\mathrm{kg} \mathrm{ha}^{-1}$ de $\mathrm{N}$ e $75 \mathrm{~kg} \mathrm{ha}^{-1}$ de $\mathrm{K}_{2} \mathrm{O}$, respectivamente.

Existe um efeito significativo e complementar na absorção de $\mathrm{N}$ e $\mathrm{K}$, que mostra que o incremento em produtividade a ser alcançado com a adubação nitrogenada depende do suprimento adequado de potássio (Silva Junior, 1987; Resende et al. (1997). No presente trabalho, observou-se que ao duplicar a dose de potássio de 90 para $180 \mathrm{~kg} \mathrm{ha}^{-1}$, reduz em apenas 3,6\% a necessidade de $\mathrm{N}\left(6,4 \mathrm{~kg} \mathrm{ha}^{-1} \mathrm{~N}\right)$ para a cultura, justificando a dose de $90 \mathrm{~kg} \mathrm{ha}^{-1}$ de $\mathrm{K}_{2} \mathrm{O}$ como 


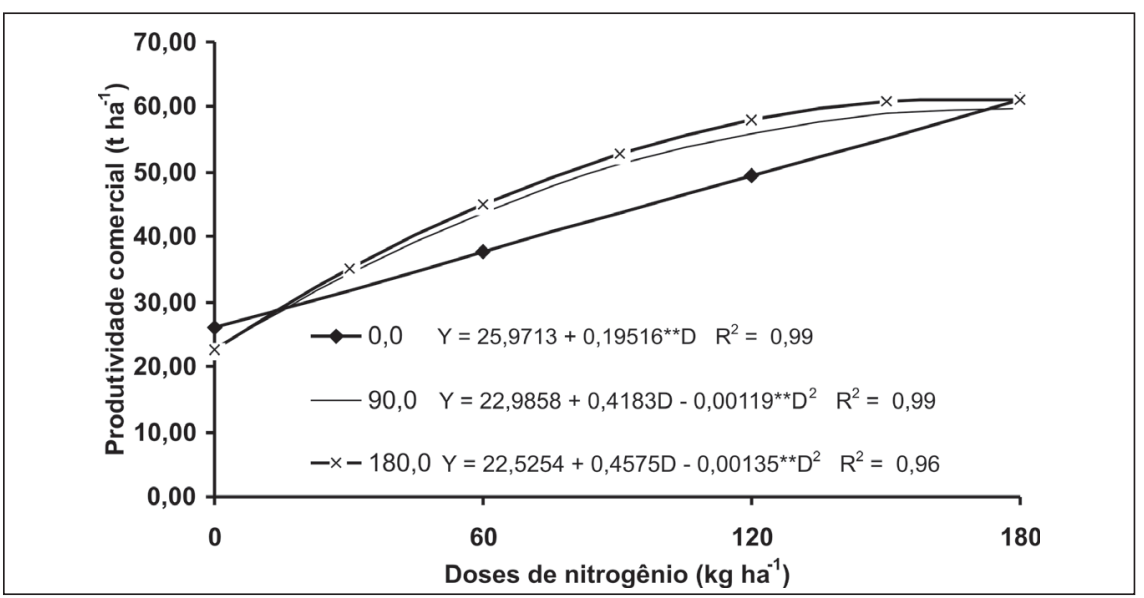

Figura 1. Produtividade comercial de bulbos de cebola nas doses 0,0; 90,0 e 180,0 kg ha-1 de potássio em função das doses de nitrogênio(Commercial yield of onion bulbs on the levels $0.0 ; 90.0$ and $180.0 \mathrm{~kg} \mathrm{ha}^{-1}$ at potassium in response to the nitrogen levels). Petrolina, Embrapa Semi-Árido, 2000.

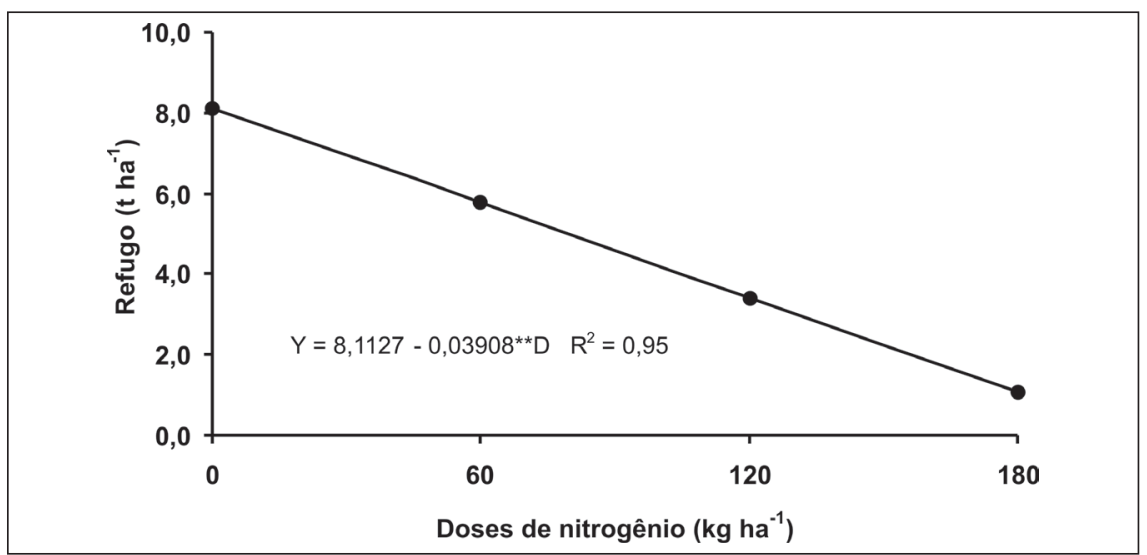

Figura 2. Produtividade não comercial de bulbos de cebola (refugos) em função das doses de nitrogênio (Non-commercial yield of onion bulbs (culls) in response to the nitrogen levels). Petrolina, Embrapa Semi-Árido, 2000.

a mais adequada nas condições estudadas (Figura 1).

No que se refere à produção de refugos (não comercial) verificou-se efeitos apenas para doses de nitrogênio, observando redução gradativa da produção de bulbos considerados não comerciais (Figura 2). Estes resultados mostram resposta da cebola à aplicação de nitrogênio e sustenta as afirmações de diferentes autores que relatam que o elemento contribui marcadamente para uma melhor produtividade da cultura, sobretudo, na produção de bulbos de maior tamanho. Resultados obtidos por May (2006) com aplicação de nitrogênio $\left(115 \mathrm{~kg} \mathrm{ha}^{-1}\right.$ de $\left.\mathrm{N}\right)$ e potássio (150 $\mathrm{kg} \mathrm{ha}^{-1}$ de $\mathrm{K}_{2} \mathrm{O}$ ), também promoveram reduções na quantidade de bulbos considerados não comerciais (refugos).
Observou-se efeitos da interação nitrogênio e potássio na massa fresca do bulbo (Figura 3). Na ausência da adubação potássica obteve-se efeito linear em função das crescentes doses de nitrogênio. $\mathrm{O}$ aumento das doses de potássio de 90 para $180 \mathrm{~kg} \mathrm{ha}^{-1}$ não exigiu proporcionalmente maior dose de nitrogênio, que foi estimada pela equação de regressão em 153,3 $\mathrm{kg} \mathrm{ha}^{-1}$. Singh et al. (2004) observaram incrementos na massa fresca do bulbo até as doses de $150 \mathrm{~kg} \mathrm{ha}^{-1}$ de $\mathrm{Ne} 120 \mathrm{~kg} \mathrm{ha}^{-1}$ de $\mathrm{K}_{2} \mathrm{O}$. Assim como para produtividade comercial de bulbos, a variação das doses de potássio não promoveu diferenças em termos da aplicação de nitrogênio para massa fresca, portanto, infere-se ser a dose de $90 \mathrm{~kg} \mathrm{ha}^{-1} \mathrm{a}$ mais adequada para as condições de realização do presente trabalho.
A classificação de bulbos comerciais de cebola foi influenciada pelas doses de nitrogênio (Tabela 1), sendo obtidos apenas bulbos classes 2 e 3 . No que se refere à classificação de bulbos classe 2 , os dados relativos a doses de nitrogênio foram ajustados a um modelo quadrático, no qual a dose de $147,3 \mathrm{~kg}$ $\mathrm{ha}^{-1}$ de nitrogênio propiciou a menor porcentagem de bulbos nesta classe $(13,3 \%)$. Esta dose de $\mathrm{N}$ é inferior à de $100 \mathrm{~kg} \mathrm{ha}^{-1}$ obtida por May (2006) para redução na distribuição de bulbos dessa classe a valores inferiores a $30 \%$. Para bulbos maiores classe 3 ( $>50$ até $70 \mathrm{~mm}$ ) também ajustou-se um modelo quadrático, no qual a dose $153,6 \mathrm{~kg} \mathrm{ha}^{-1}$ de nitrogênio promoveu a maior porcentagem de bulbos nesta característica, ou seja, $85,8 \%$. Mohanty \& Das (2001) verificaram que a produção de bulbos maiores foram obtidas com as doses de $90 \mathrm{~kg} \mathrm{ha}^{-1}$ de $\mathrm{N}$ e $60 \mathrm{~kg} \mathrm{ha}^{-1}$ de $\mathrm{K}_{2} \mathrm{O}$. Estes resultados corroboram as afirmações de Maier et al. (1990) que relatam ser o nitrogênio de grande importância para o aumento de bulbos comercialmente desejáveis, dos quais, segundo Silva et al. (1991), os de classe 3 têm a preferência do mercado consumidor nacional.

Para perda de massa, não se constatou efeitos significativos aos 20 dias (variações de 0,3 a 1,4\%), evidenciando-se apenas aos 40 e 60 dias após cura (DAC), onde as doses de nitrogênio e potássio agiram de forma independente (Tabela 1). Com relação à porcentagem de perda de massa aos 40 dias observou-se resposta quadrática na qual a dose de $142,5 \mathrm{~kg} \mathrm{ha}^{-1}$ de $\mathrm{N}$ proporcionou menor porcentagem de perda de massa de bulbos comerciais, ou seja, doses menores de nitrogênio registraram maiores perdas, verificando-se com o aumento das doses de potássio um incremento linear negativo. Resultados similares foram observados para perda de massa aos 60 dias, onde as doses de $78,9 \mathrm{~kg} \mathrm{ha}^{-1}$ de $\mathrm{N}$ e 73,8 $\mathrm{kg} \mathrm{ha}^{-1}$ de $\mathrm{K}_{2} \mathrm{O}$ promoveram as maiores perdas. Estes resultados assemelham-se aos obtidos por Singh \& Dhankhar (1991), que verificaram em bulbos armazenados sob condições ambientais que as doses de $80 \mathrm{~kg} \mathrm{ha}^{-1}$ de $\mathrm{N}$ e $100 \mathrm{~kg} \mathrm{ha}^{-1}$ de $\mathrm{K}_{2} \mathrm{O}$ tiveram as melhores respostas em qualidade de armazenamento. Sá et al. 
(2004) verificaram até 45 dias após a colheita na ausência da adubação nitrogenada perda de $21,4 \%$ enquanto que com a dose de $180 \mathrm{~kg} \mathrm{ha}^{-1}$ houve apenas $18,5 \%$ de perda.

A área utilizada para o experimento apresentou solo com teor de $\mathrm{K}=0,25$ cmol dm${ }^{-3}$, o que é considerado mediano e, de acordo com Cavalcanti (1998), necessitaria de incorporação de $90 \mathrm{~kg}$ $\mathrm{ha}^{-1}$ de $\mathrm{K}_{2} \mathrm{O}$. Os resultados obtidos indicaram ser esta a dose mais adequada quando associada (em interação) com $\mathrm{a}(\mathrm{s})$ dose(s) de nitrogênio, que proporcionaram maior produtividade comercial e massa fresca de bulbos.

Os resultados obtidos permitem concluir que a produtividade comercial de cebola aumentou linearmente com as doses de $\mathrm{N}$ na ausência da adubação potássica, enquanto as doses de 90 e 180 $\mathrm{kg} \mathrm{ha}^{-1}$ de $\mathrm{K}_{2} \mathrm{O}$ proporcionaram máximas produtividades nas doses de 175,8 e $169,4 \mathrm{~kg} \mathrm{ha}^{-1}$ de N. A produção de refugos (bulbos não comerciais) reduziu linearmente com o aumento das doses de nitrogênio. A massa fresca do bulbo cresceu linearmente na ausência da adubação potássica, enquanto para as doses de 90 e $180 \mathrm{~kg} \mathrm{ha}^{-1}$ de $\mathrm{K}_{2} \mathrm{O}$ o maior rendimento de massa fresca do bulbo foi obtido na dose de $153,3 \mathrm{~kg} \mathrm{ha}^{-1}$ de N. A classificação de bulbos comerciais de cebola foi influenciada pelas doses de nitrogênio, sendo que a maior porcentagem de bulbos da classe 3 obtido $(85,8 \%)$ correspondeu à dose de 153,6 $\mathrm{kg} \mathrm{ha}^{-1}$ de $\mathrm{N}$. As doses de nitrogênio e potássio agiram de forma independente aos 40 e 60 dias após cura reduzindo as perdas pós-colheita.

\section{REFERÊNCIAS}

BALOCH MA; BALOCH AF; BALOCH G; ANSARI AH; QAYYUM SM. 1991. Growth and yield response of onion to different nitrogen and potassium fertilizer combination levels. Sarhad Journal Agriculture 7: 63-66.

BRASIL. 1995. Ministério da Agricultura, Abastecimento e Reforma Agrária. Diário Oficial da República Federativa do Brasil. Brasília, Seção 1, p.13513. (Portaria n. 529 de 18 agosto 1995).

CAVALCANTI FJA. 1998. Recomendações de adubação para o Estado de Pernambuco: $2^{\mathrm{a}}$ aproximação. Recife: IPA. 198p.

DIAZ-PEREZ JC; PURVIS AC; PAULK JT. 2003. Bolting, yield, and bulb decay of sweet onion as affected by nitrogen fertilization. Journal American Society Horticultural Science 128: 144-149.

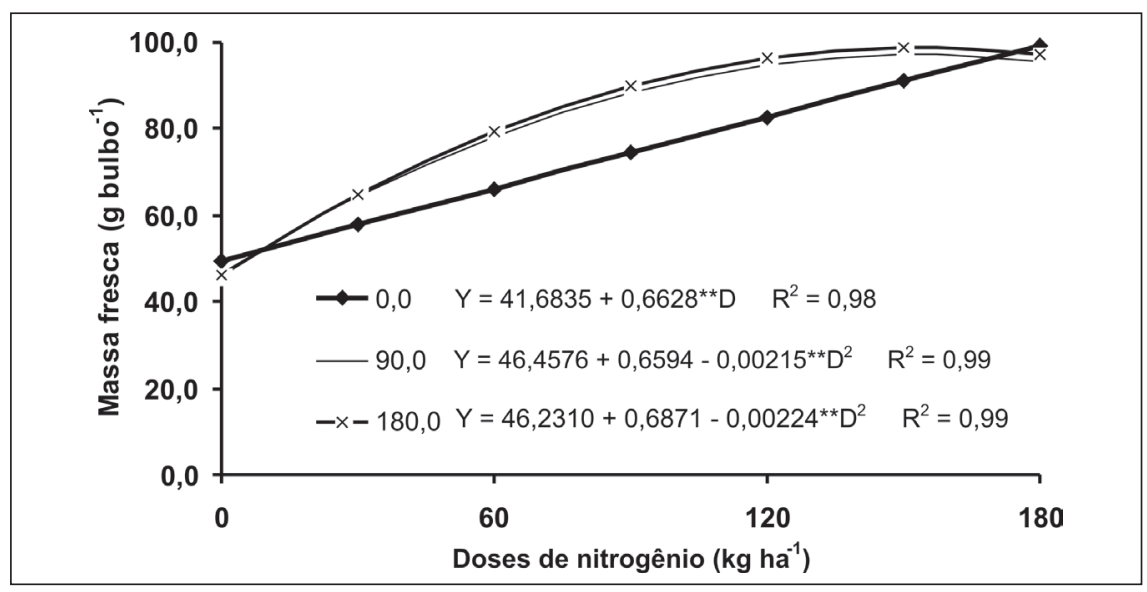

Figura 3. Massa fresca do bulbo de cebola nas doses 0,0; 90,0 e 180,0 $\mathrm{kg} \mathrm{ha}^{-1}$ de potássio em função das doses de nitrogênio (Fresh mass of onion bulbs on the levels $0.0 ; 90.0$ and $180.0 \mathrm{~kg}$ $\mathrm{ha}^{-1}$ at potassium in response to the nitrogen levels). Petrolina, Embrapa Semi-Árido, 2000.

Tabela 1. Equações de regressão para classificação, em classes (\%), segundo o diâmetro transversal e porcentagem de perda de peso aos 40 e 60 dias após a cura (DAC) de bulbos de cebola de doses $(\mathrm{D})$ de potássio $(\mathrm{K})$ e nitrogênio $(\mathrm{N})$ (Regression equations for classification in class (\%), second the transversal diameter and percentage of loss of weight to 40 and 60 days after the cure (DAC) of onion bulbs to the potassium (K) and nitrogen (N) levels (D)). Petrolina, Embrapa Semi-Árido, 2000.

\begin{tabular}{lll}
\hline Características & Equações de regressão & \\
\hline Classe 2 & $\mathrm{~N}: \mathrm{Y}=54,9389-0,4743 \mathrm{D}+0,00161^{* *} \mathrm{D}^{2}$ & $\mathrm{R}^{2}=0,99$ \\
Classe 3 & $\mathrm{~N}: \mathrm{Y}=38,7073+0,3932 \mathrm{D}-0,00128^{* *} \mathrm{D}^{2}$ & $\mathrm{R}^{2}=0,99$ \\
\multirow{2}{*}{$40 \mathrm{DAC}$} & $\mathrm{N}: \mathrm{Y}=13,8947-0,0513 \mathrm{D}+0,00018^{* *} \mathrm{D}^{2}$ & $\mathrm{R}^{2}=0,97$ \\
& $\mathrm{~K}: \mathrm{Y}=12,2904-0,0081^{*} \mathrm{D}$ & $\mathrm{R}^{2}=0,97$ \\
$60 \mathrm{DAC}$ & $\mathrm{N}: \mathrm{Y}=15,4405+0,0300 \mathrm{D}-0,00019^{* *} \mathrm{D}^{2}$ & $\mathrm{R}^{2}=0,87$ \\
& $\mathrm{~K}: \mathrm{Y}=15,7583+0,0118 \mathrm{D}-0,00008^{*} \mathrm{D}^{2}$ & $\mathrm{R}^{2}=0,99$ \\
\hline
\end{tabular}

*;**Significativo ao nível de 5 e $1 \%$ de probabilidade, pelo teste de $\mathrm{F}$.

EMBRAPA - EMPRESA BRASILEIRA DE PESQUISA AGROPECUÁRIA. Sistema Brasileiro de Classificação de Solos. Brasília: Embrapa Produção de Informações (SPI), 1999. $412 \mathrm{p}$.

FAO. 2007. Agricultural production, primary crops. Disponível em http://www.fao.org. Acessado em 26 de fevereiro de 2007.

FARIAS CM; PEREIRA JR. 1992. Fontes e níveis de nitrogênio na produtividade da cebola no Vale do São Francisco. Pesquisa Agropecuária Brasileira 27: 403-407.

FILGUEIRA FAR. 2003. Novo manual de olericultura: agrotecnologia moderna produção e comercialização de hortaliças. 2 . ed., Viçosa: Editora UFV. 412p.

GOMES FP. 2000. Curso de estatística experimental. 14.ed. São Paulo: Nobel. 477p.

INSTITUTO BRASILEIRO DE GEOGRAFIA E ESTATÍSTICA - IBGE. 2006. Levantamento Sistemático da Produção Agrícola. Rio de Janeiro: IBGE. v.18. p.1-76.

LEE JT; HA IJ; LEE CJ; MOON JS; CHO YC. 2003. Effect of $\mathrm{N}_{1} \mathrm{P}_{2} \mathrm{O}_{5}$, and $\mathrm{K}_{2} \mathrm{O}$ application rates and top dressing time on growth and yield of onion (Allium cepa $\mathrm{L}$.) under spring culture in low land. Korean Journal Horticultural Science and Technology 21: 260-266.
MAIER NA; DAHLENBURGAP; TWIGDENTK. 1990. Effect of nitrogen on the yield and quality of irrigated onions (Allium cepa L.) cv. Cream Gold grown on siliceous sands. Australian Journal of Experimental Agriculture 30: 845-851.

MANDIRAC; KHANAH. 2003. Effect of nitrogen and potassium on growth, yield and yield attributes of onion. New Agriculturist 14: 9-11.

MAY A. 2006. Desempenho de híbridos de cebola em função da população de plantas $e$ fertilização nitrogenada e potássica. Jaboticabal: UNESP-FCAV. 142p (Tese doutorado).

MOHANTY BB; DAS JN. 2001. Response of rabi onion cv. Nasik Red to nitrogen and potassium fertilization. Vegetable Science 28: 40-42.

RESENDE GM; SILVA GL; PAIVA LE; DIAS PF; CARVALHO JG. 1997. Resposta do milho (Zea mays L.) a doses de nitrogênio e potássio em solo da região de Lavras-MG. II. Macronutrientes na parte aérea. Ciência e Agrotecnologia 21: 477-483.

SÁ GD; BARTHG; REGHIN MY; CAIRES EF. 2004. Influência de doses e épocas de aplicação de nitrogênio na produção de pós-colheita da cultura da cebola. In: CONGRESSO BRASILEIRO DE OLERICULTURA, 44. Horticultura Brasileira 22: Suplemento CD-ROM. 
SHER DJ. Fertilizers for onions. 1996. Commercial Grower 51: 12-13.

SHUKLA V; RAO KPGK; PRABHAKAR BS. 1992. Effect of nitrogen on bulb yield and keeping quality of onion cultivars. Progressive Horticulture 21: 244-245.

SILVA E; TEIXEIRA LAJ; AMADO TJC. 1991. The increase in onion production in Santa Catarina State, South Brazil. Onion Newsletter for the Tropics 3: 7-9.

SILVA JUNIOR AA. 1987. Adubação mineral e orgânica em repolho. II. Concentração de nutrientes na folha e precocidade. Horticultura Brasileira 5: 15-17.

SINGH D; SHARMA RP. 1991. Effect of soil moisture regimes and nitrogen fertilization on onion. Indian Journal of Agronomy 36: 125-126.
SINGH J; DHANKHAR BS. 1991. Effect of nitrogen, potash and zinc on storage loss of onion bulbs (Allium cepa L.). Vegetable Science 18: 16-23.

SINGH S; YADAV PK; SINGH B. 2004. Effect of nitrogen and potassium on growth and yield of onion (Allium cepa L.) cv. Pusa Red. Haryana Journal Horticultural Sciences 33: 308-309.

SINGH SP; VERMA AB. 2001. Response of onion (Allium cepa) to potassium application. Indian Journal Agronomy 46: 182-185.

SYED N; MUNIR M; ALIZAI AA; GHAFOOR A. 2000. Onion yield and yield components as function of the levels of nitrogen and potassium application. Pakistan Journal of Biological Sciences 3: 2069-2071.
SOUZA RJ; RESENDE GM. 2002. Cultura da cebola. Lavras: UFLA. 115p (Textos Acadêmicos - Olericultura, 21).

VACHCHANI MU; PATEL ZG. 1996. Growth and yield of onion (Allium cepa L.) as influenced by levels of nitrogen, phosphorus and potash under south Gujarat conditions. Progressive Horticulture 25: 166-167.

WARID WA; GUERRERO JC; LOAIZA JM. 1996. Storage quality of ten onion cultivars evaluated in Sonora, Mexico. Onion Newsletter for the Tropics 7: 17-22. 